\title{
Velocity Selective Polarization Spectroscopy for Modulation-Free Dispersion Signals at Detuned Frequencies
}

\author{
Hoon Yu, Seung Jin Kim, and Jung Bog Kim \\ Korea National University of Education, Chungbuk 363-791, Republic of Korea \\ Correspondence should be addressed to Jung Bog Kim; jbkim@knue.ac.kr
}

Received 23 October 2014; Accepted 8 December 2014; Published 30 December 2014

Academic Editor: Masaki Oura

Copyright (c) 2014 Hoon Yu et al. This is an open access article distributed under the Creative Commons Attribution License, which permits unrestricted use, distribution, and reproduction in any medium, provided the original work is properly cited.

\begin{abstract}
We present a simple technique to obtain modulation-free locking signals at the detuned frequency from an atom transition between hyperfine structures. Polarization spectroscopy allows us to obtain the dispersion signals that are suitable for frequency locking. Velocity selective optical pumping using another laser beam also allows us to obtain signals at the detuned frequency and to shift crossover signal away. By combining these two techniques, we were able to obtain the velocity selective birefringence signal only at the principle transition in an $\mathrm{Rb}$ vapor cell and compare the birefringence signal with the theoretical spectrum predicted by using Nakayama's model.
\end{abstract}

\section{Introduction}

Long-term stabilization of a laser frequency is an essential part for many applications in atom, molecular, and optical physics. Error signal for frequency stabilization is typically achieved by using dispersion or dispersion-like spectra because these spectra are zero-crossing at the atom transition frequency and have opposite charge on either side of the frequency fluctuation. The most popular way to obtain the dispersion signal is through phase-sensitive detection with frequency modulation spectroscopy $[1,2]$, by which the first derivative of absorption profiles can be obtained. However, the frequency bandwidth of the locked laser could be limited by both the modulation ranges in frequency and the time constant of a lock-in phase-sensitive detector. Techniques which are based on both birefringence and dichroism are alternatively used for modulation-free frequency stabilization. Birefringence of medium employs a dispersion signal by polarization spectroscopy [3-5]. Dichroism based on transition among the sub-Zeeman levels is used to obtain the dispersion-like signal [6-9]. These two techniques allow us to lock a laser frequency without frequency modulation on the atom transition.
Detuned laser beams are useful in some cases although the resonant beam on atom transition strongly interacts with atoms. For example, laser cooling [10] and polarization gradient cooling $[11,12]$ are performed in red-detuning of few tens megahertz. Stimulated Raman transition using few hundreds or thousands megahertz detuned beams to make atom interferometer is a representative method for reducing decoherent effects caused by spontaneous emission [13, 14]. Also, a very far detuned laser beam is useful to generate appropriate potential for optical trap of neutral atoms or molecules $[15,16]$. In most cases, acoustooptic or electrooptic modulators are used for a certain detuning. These modulators can also generate error signals that decline the accuracy of measurement. Recently, Duan et al. [17] reported the novel method to obtain a saturated absorption spectrum at high sensitivity with displaced crossovers. This idea is very good because effects by crossover signals can be removed when accurate atom transition frequency is measured.

In this paper, we demonstrate a simple method to obtain birefringence signal at detuned frequency from resonance without any modulator. We adopted polarization spectroscopy to obtain the birefringence signal by measuring the rotation of the probe beam's polarization due to the pumping 


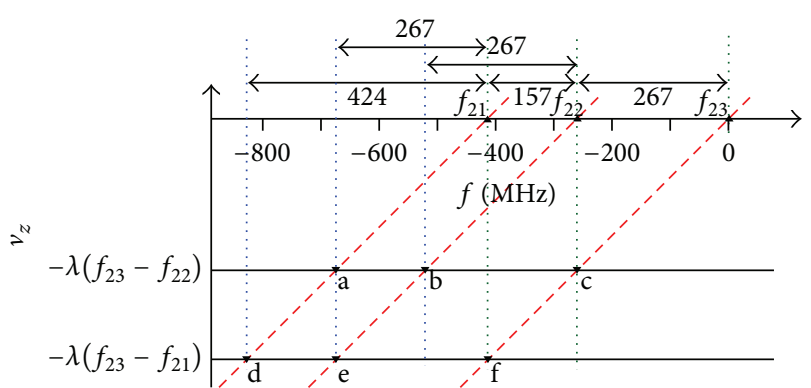

FIGURE 1: Scheme for VSPS where the pump beam is frequency locked on the $5 \mathrm{~S}_{1 / 2}(F=2) \rightarrow 5 \mathrm{P}_{3 / 2}\left(F^{\prime}=3\right)$ and probe beam is swept over the range of the $5 \mathrm{~S}_{1 / 2}(F=2) \rightarrow 5 \mathrm{P}_{3 / 2}$ transition. Triangles represent an absorption peak due to the zero velocity component and reverse triangles represent the nonzero velocity component.

beam in an $\mathrm{Rb}$ vapor cell and the spectra are compared to the Nakayama's theory based on low laser intensity [18-20]. Furthermore, we can eliminate effect of crossover signals by using frequency stabilized pumping that oppositely propagate to probe beam and generate velocity selective signals. These birefringence signals with displaced crossovers could be good for locking the probe laser frequency.

\section{Nakayama Theory for Velocity Selective Birefringence Model}

Nakayama considered the population change of each magnetic sublevel by considering the absorption and emission of light in a single cycle of an optical pumping [18, 19]. Since this model assumed a weak light intensity, Nakayama theory is not able to predict coherent effects such as EIT (Electromagnetically Induced Transparency) and EIA (Electromagnetically Induced Absorption). Nevertheless, it is a well-known method for predicting the line profile of the absorption spectrum of an optical pumping, a polarization spectroscopy, and a dichroism in weak intensity regimes [20]. In the Nakayama scheme, two laser beams are typically considered. The first laser, called the pumping beam, redistributes atom population in magnetic sublevels after an interaction with the atoms. The second beam, called the probe beam, is used to obtain a spectrum by measuring its susceptibility in the redistributed atom medium.

Figure 1 shows the diagram for one of the schemes for our velocity selective polarization spectroscopy (VSPS) where the pumping beam is frequency locked on the $5 \mathrm{~S}_{1 / 2}(F=2) \rightarrow$ $5 \mathrm{P}_{3 / 2}\left(F^{\prime}=3\right)$ resonance of the ${ }^{87} \mathrm{Rb}$ atom transition and the frequency of the probe beam is swept over the range of $5 \mathrm{~S}_{1 / 2}(F=2) \rightarrow 5 \mathrm{P}_{3 / 2}$ transition. From the atomic frame, there are three velocity groups that see the pumping beam resonant due to the Doppler effect in horizontal line in Figure 1. For probe beam, the resonant signals in the velocityfrequency regime are described with diagonal lines as shown by the red dashed line in Figure 1 [17]. Velocity selective absorption peaks are observed at the point of intersections in

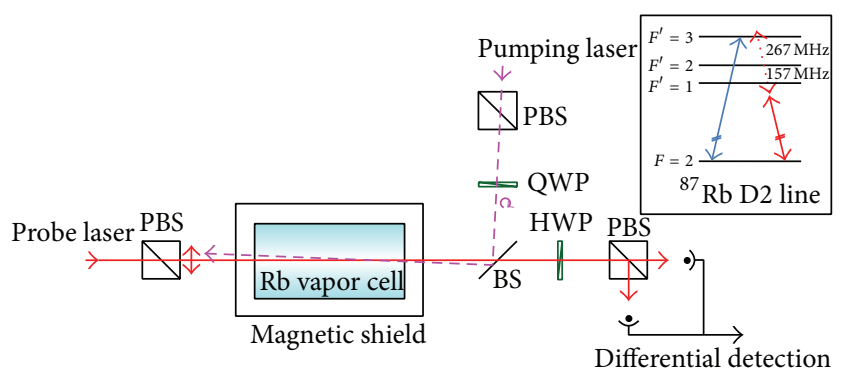

FIGURE 2: Schematic diagram of experimental setup and hyperfine structure of ${ }^{87} \mathrm{Rb}$ (color online) (PBS: polarization beam splitter, BS: beam splitter, QWP: quarter wave plate, and HWP: half wave plate).

Figure 1. Theoretical VSPS signal based on Nakayama theory is given by

$$
\begin{aligned}
\operatorname{VSPS}(\Delta)= & \sum_{i=1,2,3} S_{2 i} L_{r e}\left(\frac{2 \Delta_{2 i}^{23}}{\gamma}\right) \\
& +\sum_{i=1,2,3} V_{2 i}^{22} L_{r e}\left(\frac{2 \Delta_{23-22}^{2 i}}{\gamma}\right) \operatorname{Exp}\left[-\left(\frac{\Delta_{22}^{23}}{k u}\right)^{2}\right] \\
& +\sum_{i=1,2,3} V_{2 i}^{21} L_{r e}\left(\frac{2 \Delta_{23-21}^{2 i}}{\gamma}\right) \operatorname{Exp}\left[-\left(\frac{\Delta_{21}^{23}}{k u}\right)^{2}\right],
\end{aligned}
$$

where $S_{2 i}$ is the transition strength [20] for $F=2 \rightarrow F^{\prime}=i$ transition, $V_{2 i}^{22}$ is the transition strength for the crossover peak between $F=2 \rightarrow F^{\prime}=2$ and $F=2 \rightarrow F^{\prime}=i$, and $\Delta_{2 i}^{23}$ is the detuning from $F=2 \rightarrow F^{\prime}=3$ transition to $F=2 \rightarrow$ $F^{\prime}=i$ and $u=\sqrt{2 k_{B} T / M} \cdot L_{r e}$ is the line shape function given by

$$
L_{r e}(\Delta)=\frac{s_{0}}{\sqrt{1+s_{0}}} \frac{2 \Delta}{4 \Delta^{2}+\left(1+\sqrt{1+s_{0}}\right)^{2}},
$$

where $s_{0}$ is the normalized beam intensity relative to the saturation intensity. Each term in the right side of (1) represents the case of zero, $v_{1}$ and $v_{2}$ velocity component.

\section{Experimental Setup and Result}

Figure 2 shows our experimental setup for obtaining birefringence signals. We use two external cavity diode lasers (ECDL). The laser for the pumping beam is frequency locked on the resonant transition or crossover peak in D2 transition of ${ }^{87} \mathrm{Rb}$ atoms by using a conventional saturated absorption spectroscopy with frequency modulation. The frequency of the probe beam is swept over the range of the $5 \mathrm{~S}_{1 / 2}(F=2) \rightarrow$ $5 \mathrm{P}_{3 / 2}$ transition. Powers of the probe and pumping beams are $370 \mu \mathrm{W}$ and $290 \mu \mathrm{W}$, respectively. The probe laser has a circular shape with $0.75 \mathrm{~mm}$ diameter while the pumping beam has a rectangular shape with $0.75 \mathrm{~mm} \times 1.3 \mathrm{~mm}$. The $\mathrm{Rb}$ cell is $10 \mathrm{~cm}$ long. It was kept at room temperature of $23^{\circ} \mathrm{C}$ 


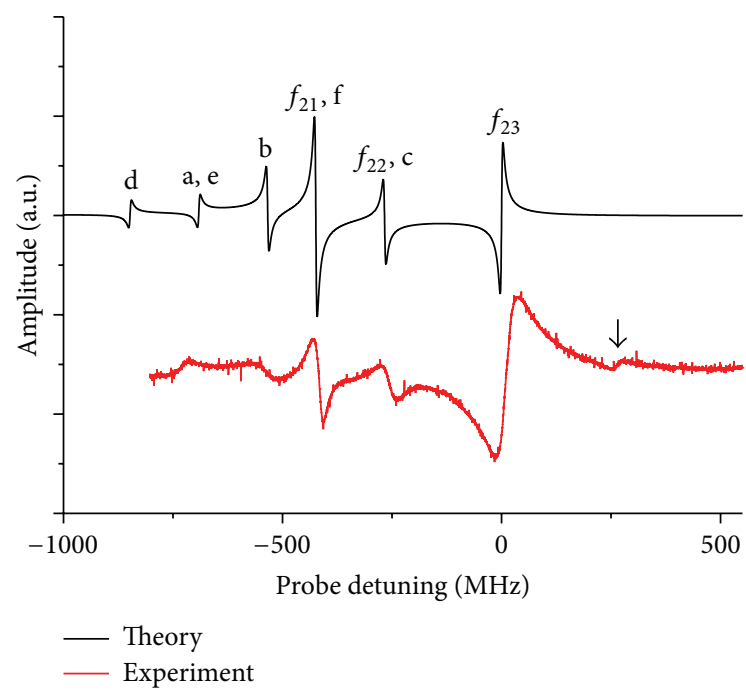

FIGURE 3: Velocity selective polarization spectrum as probe detuning from $5 \mathrm{~S}_{1 / 2}(F=2) \rightarrow 5 \mathrm{P}_{3 / 2}\left(F^{\prime}=3\right)$ transition frequency for the case of the laser frequency configuration in Figure 1.

and wrapped in 3 turns with $\mu$-metal sheet for shielding earth magnetic field.

The linear polarization of the probe beam can be treated to the summation of two opposite types of circular polarization where the pumping beam is circularly polarized. Refractive indexes and absorption coefficients for $\sigma^{+}$and $\sigma^{-}$ component of the probe laser become different in polarized atoms, called birefringence and dichroism, respectively. Optical birefringence induces rotating the polarization axis of probe laser field by $\Delta \theta=\Delta n k_{0} L / 2$ [2], where $k_{0}$ is the wave number of the probe laser in vacuum and $L$ is the length of atom vapor sample. To measure the rotation of probe beam's polarization, we put a half wave plate and a polarization beam splitter in front of two detectors. We can set the difference between two detectors to zero by rotating the half wave plate without the pumping beam. Then, we can measure the rotation of the probe field due to birefringence after passing through the atom sample. The probe and the pump laser beams are slightly misaligned in order to avoid fluctuation by feedback to the laser cavity.

Figure 3 shows the experimental VSPS and theoretical curve from (1) for the laser frequency configuration in Figure 1. It is clear that the crossover peaks are shifted away. In a conventional polarization spectroscopy, a pumping beam and a counterpropagating probe beam come from the same laser, so they always have the same frequency in the laboratory frame. When two frequencies in the atomic frame are resonant with other transitions due to the Doppler effect, the crossover peak is observed in the middle of the two resonant frequencies. With two lasers prepared independently, however, the crossover can be shifted away and velocity selective signals appear. Even though both the crossover and the velocity selective signal are based on Doppler effect, the key points of the velocity selective signal are that it is possible to shift the crossover peak and change the peak position by changing pumping beam's locking frequency. Moving the crossover peak position away enhances the signal sensitivity [17]. It is also worthy to note that VSPS gives us a signal similar to a cold atom [21].

The signal sensitivity for the transition $5 \mathrm{~S}_{1 / 2}(F=2) \rightarrow$ $5 \mathrm{P}_{3 / 2}\left(F^{\prime}=1\right)$ is relatively low due to its low transition strength for conventional polarization spectroscopy, 0.03 times of $5 \mathrm{~S}_{1 / 2}(F=2) \rightarrow 5 \mathrm{P}_{3 / 2}\left(F^{\prime}=3\right)$ transition for ${ }^{87} \mathrm{Rb}$ [20]. However, in the VSPS scheme, the contribution of the moving atom marked as "f" in Figures 1 and 3 increases the signal sensitivity so that a comparable size with the strongest transition was shown in Figure 3, theoretically and experimentally. The signal of "d" in theoretical spectrum was not observed because of the scan limit of probe beam's frequency in our laser system.

Figure 4 shows how the VSPS changes as frequency of the pumping beam was changed. Pumping beam is locked on the crossover peak between $5 \mathrm{~S}_{1 / 2}(F=2) \rightarrow 5 \mathrm{P}_{3 / 2}\left(F^{\prime}=3\right)$ and $5 \mathrm{~S}_{1 / 2}(F=2) \rightarrow 5 \mathrm{P}_{3 / 2}\left(F^{\prime}=2\right)$ transitions for (a) and (d) in Figure 4. Since the pumping beam has a nonresonant frequency, all signals are on detuned frequencies from atom resonance. The energy difference between atom hyperfine structures determines the detuning. In our case, signals are on $-714.5,557.5,-400.5,-290.5,-133.5$, and $133.5 \mathrm{MHz}$ from the $5 \mathrm{~S}_{1 / 2}(F=2) \rightarrow 5 \mathrm{P}_{3 / 2}\left(F^{\prime}=3\right)$ transition as shown in (a) and $(\mathrm{d})$. The pumping beam was locked on $5 \mathrm{~S}_{1 / 2}(F=2) \rightarrow$ $5 \mathrm{P}_{3 / 2}\left(F^{\prime}=3\right)$ transition for $(\mathrm{b})$ and $(\mathrm{e})$ and crossover peak between $5 \mathrm{~S}_{1 / 2}(F=2) \rightarrow 5 \mathrm{P}_{3 / 2}\left(F^{\prime}=3\right)$ and $5 \mathrm{~S}_{1 / 2}(F=2) \rightarrow$ $5 \mathrm{P}_{3 / 2}\left(F^{\prime}=1\right)$ transitions for $(\mathrm{c})$ and $(\mathrm{f})$. Signals are on -581 , $-424,-267,-157,0$, and $267 \mathrm{MHz}$ for (b) and (e). And for (c) and (f), signals are on $-636,-479,-322,-212,-55$, and $212 \mathrm{MHz}$. VSPS positions depend on the pumping beam's frequency and nonresonant pumping beam makes various detuning for VSPS as shown in Figure 4. Unknown signals which are not expected from our diagram in Figures 1 and 4(b) are observed, marked in black arrow in Figures 3 and 4(e), which should be identified.

The slope of the experimental VSPS signals matches well with the Nakayama theory. It means that we are not in the strong field regime which can induce coherence effect like EIT or EIA even though there are V and I type configurations.

\section{Conclusion}

We were able to obtain the dispersion spectra without the frequency modulation by using a polarization spectroscopy using two independent laser sources in an ${ }^{87} \mathrm{Rb}$ vapor cell. The velocity selective polarization spectra were obtained on the detuned frequencies which related the frequency difference between atomic resonances as the pumping beam's frequency. Since crossover peaks were also moved away from the around of principle signals, we were able to increase the sensitivity of principle peak's signal. Furthermore, the low signal size of the weak transition for instance $5 \mathrm{~S}_{1 / 2}(F=2) \rightarrow 5 \mathrm{P}_{3 / 2}\left(F^{\prime}=1\right)$ can be increased by adding a moving atom effect along the beam's propagating direction. The technique of the velocity 


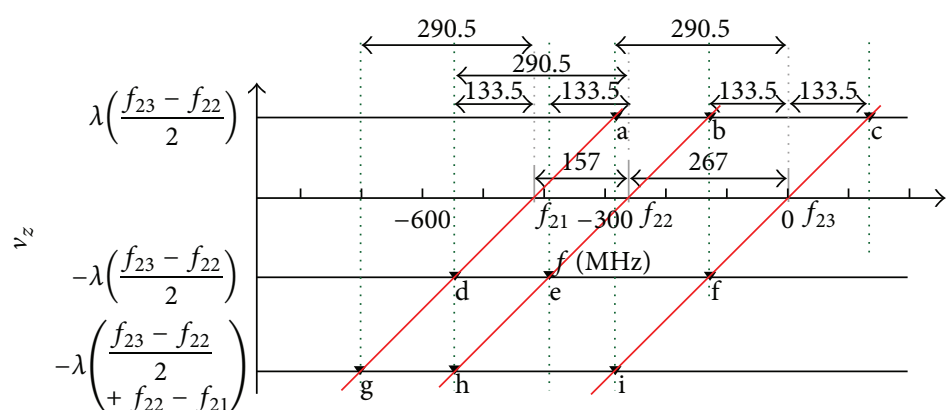

(a)

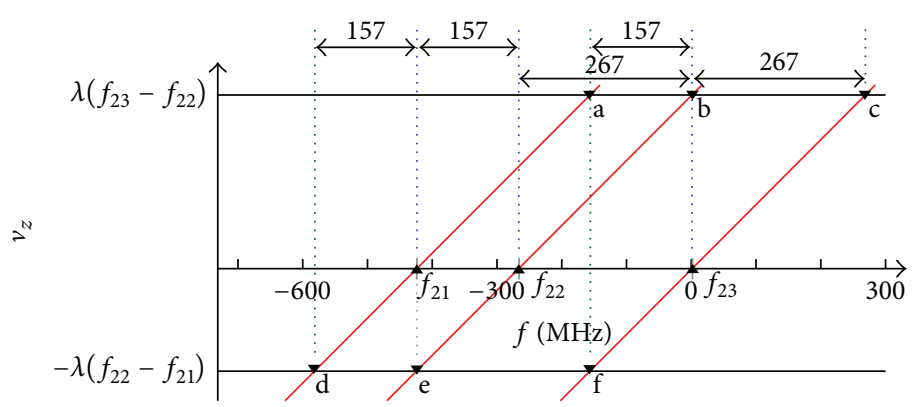

(b)

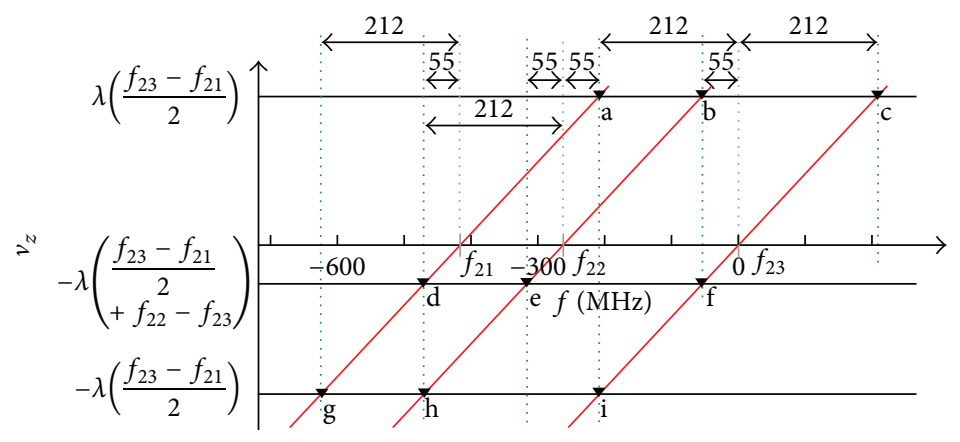

(c)

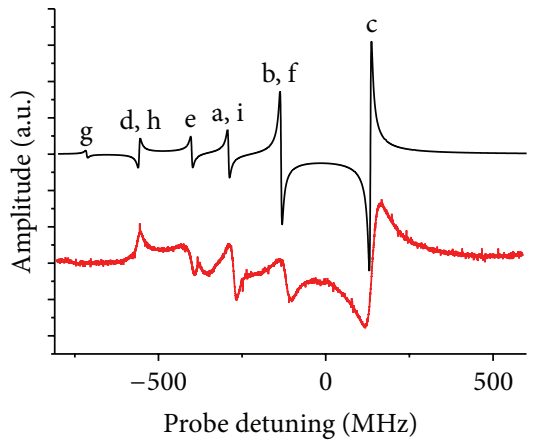

(d)

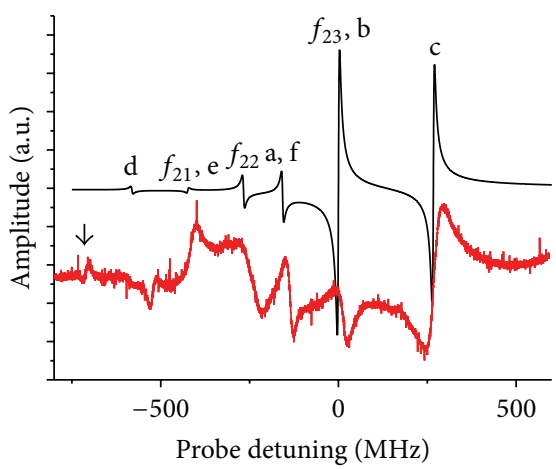

(e)

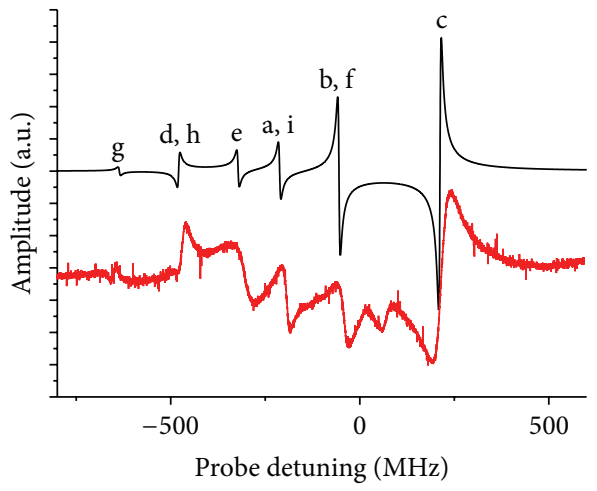

(f)

FIgURE 4: VSPS as a pumping beam's frequency. The pumping beam is locked on the crossover peak between $F=2 \rightarrow F^{\prime}=3$ and $F=2 \rightarrow F^{\prime}=2$ transition for (a) and (d), $F=2 \rightarrow F^{\prime}=2$ resonant peak for (b) and (e), and crossover peak between $F=2 \rightarrow F^{\prime}=3$ and $F=2 \rightarrow F^{\prime}=1$ transition for (c) and (f). Black lines represent theoretical curves based on Nakayama theory and the red lines represent experimental results in (e)-(f).

selective polarization spectroscopy is useful to obtain the modulation-free dispersion signals at detuned frequencies from atomic resonance for laser frequency locking. Also it gives us spectra such as the signals from a Doppler-free cold atom medium.

\section{Conflict of Interests}

The authors declare that there is no conflict of interests regarding the publication of this paper.

\section{Acknowledgment}

This work was supported by the National Research Foundation of Korea (NRF) Grant funded by the Korean government (MSIP) (no. 2014R1A2A2A01007460).

\section{References}

[1] G. C. Bjorklund, "Frequency-modulation spectroscopy: a new method for measuring weak absorptions and dispersions," Optics Letters, vol. 5, no. 1, pp. 15-17, 1980. 
[2] T. Mitsui, K. Yamashita, and K. Sakurai, "Diode laser-frequency stabilization by use of frequency modulation by a vibrating mirror," Applied Optics, vol. 36, no. 22, pp. 5494-5498, 1997.

[3] J. B. Kim, H. J. Kong, and S. S. Lee, "Dye laser frequency locking to the hyperfine structure $\left(3 S_{1 / 2}, F=2-3 P_{1 / 2}, F=2\right)$ of sodium D1 line by using polarization spectroscopy," Applied Physics Letters, vol. 52, no. 6, pp. 417-419, 1988.

[4] Y. Yoshikawa, T. Umeki, T. Mukae, Y. Torii, and T. Kuga, "Frequency stabilization of a laser diode with use of lightinduced birefringence in an atomic vapor," Applied Optics, vol. 42, no. 33, pp. 6645-6649, 2003.

[5] C. Wieman and T. W. Hänsch, "Doppler-free laser polarization spectroscopy," Physical Review Letters, vol. 36, no. 20, pp. 1170$1173,1976$.

[6] M. Pichler and D. C. Hall, "Simple laser frequency locking based on Doppler-free magnetically induced dichroism," Optics Communications, vol. 285, no. 1, pp. 50-53, 2012.

[7] K. L. Corwin, Z.-T. Lu, C. F. Hand, R. J. Epstein, and C. E. Wieman, "Frequency-stabilized diode laser with the Zeeman shift in an atomic vapor," Applied Optics, vol. 37, no. 15, pp. 32953298, 1998.

[8] V. V. Yashchuk, D. Budker, and J. R. Davis, "Laser frequency stabilization using linear magneto-optics," Review of Scientific Instruments, vol. 71, no. 2, pp. 341-346, 2000.

[9] C. I. Sukenik, H. C. Busch, and M. Shiddiq, "Modulation-free laser frequency stabilization and detuning," Optics Communications, vol. 203, no. 1-2, pp. 133-137, 2002.

[10] S. Chu, L. W. Hollberg, J. E. Bjorkholm, A. Cable, and A. Ashkin, "Three-dimensional viscous confinement and cooling of atoms by resonance radiation pressure," Physical Review Letters, vol. 55 , no. 1, pp. $48-51,1985$.

[11] J. Dalibard and C. Cohen-Tannoudji, "Laser cooling below the Doppler limit by polarization gradients: simple theoretical models," Journal of the Optical Society of America B, vol. 6, no. 11, pp. 2023-2045, 1989.

[12] P. J. Ungar, D. S. Weiss, E. Riis, and S. Chu, "Optical molasses and multilevel atoms: theory," Journal of the Optical Society of America B, vol. 6, no. 11, pp. 2058-2071, 1989.

[13] M. Kasevich and S. Chu, "Atomic interferometry using stimulated Raman transitions," Physical Review Letters, vol. 67, no. 2, pp. 181-184, 1991.

[14] A. D. Cronin, J. Schmiedmayer, and D. E. Pritchard, "Optics and interferometry with atoms and molecules," Reviews of Modern Physics, vol. 81, no. 3, pp. 1051-1129, 2009.

[15] A. Ashkin, J. M. Dziedzic, J. E. Bjorkholm, and S. Chu, "Observation of a single-beam gradient force optical trap for dielectric particles," Optics Letters, vol. 11, no. 5, pp. 288-290, 1986.

[16] K. C. Neuman and S. M. Block, "Optical trapping," Review of Scientific Instruments, vol. 75, no. 9, pp. 2787-2809, 2004.

[17] J. Duan, X. Qi, X. Zhou, and X. Chen, "Detection of saturated absorption spectroscopy at high sensitivity with displaced crossovers," Optics Letters, vol. 36, no. 4, pp. 561-563, 2011.

[18] S. Yagi and K. Nakayama, "Ultrasonoecho spectral moment tomography," Japanese Journal of Applied Physics, vol. 24, no. S1, p. $177,1985$.

[19] S. Nakayama, "Optical pumping effects in high resolution laser spectroscopy," Physica Scripta, vol. 1997, no. 70, article 64, 1997.

[20] H. R. Noh, "Simple analytical theory of saturation and polarization spectroscopy of alkali-metal atoms by using Nakayama's model," Journal of the Korean Physical Society, vol. 57, p. 1381, 2010.
[21] H. Yu, K. S. Kim, J. D. Kim, H. K. Lee, and J. B. Kim, “Observation of Doppler-free electromagnetically induced transparency in atoms selected optically with specific velocity," Physical Review A, vol. 84, Article ID 052511, 2011. 

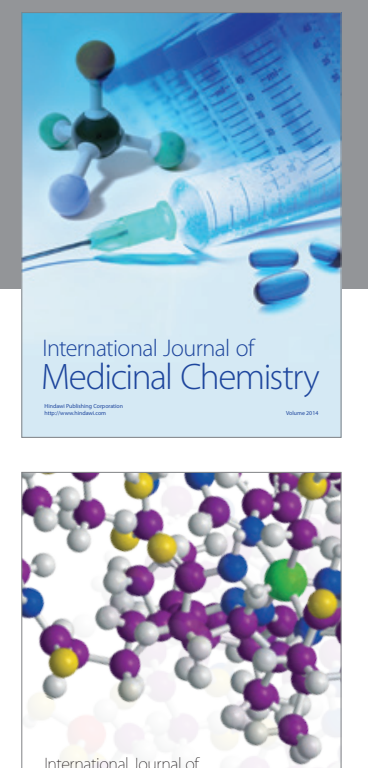

\section{Carbohydrate} Chemistry

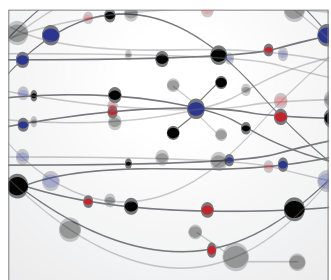

The Scientific World Journal
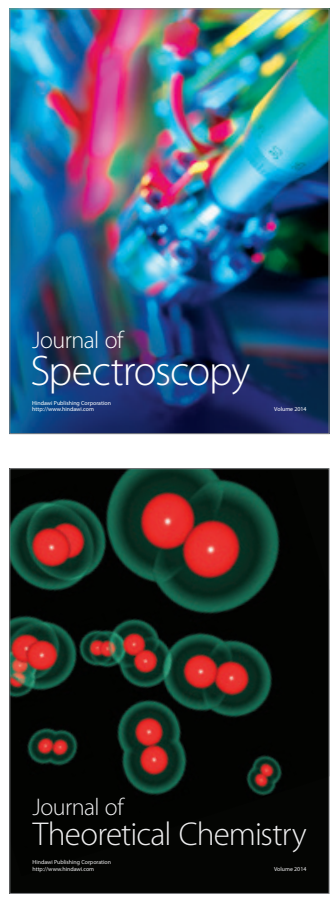
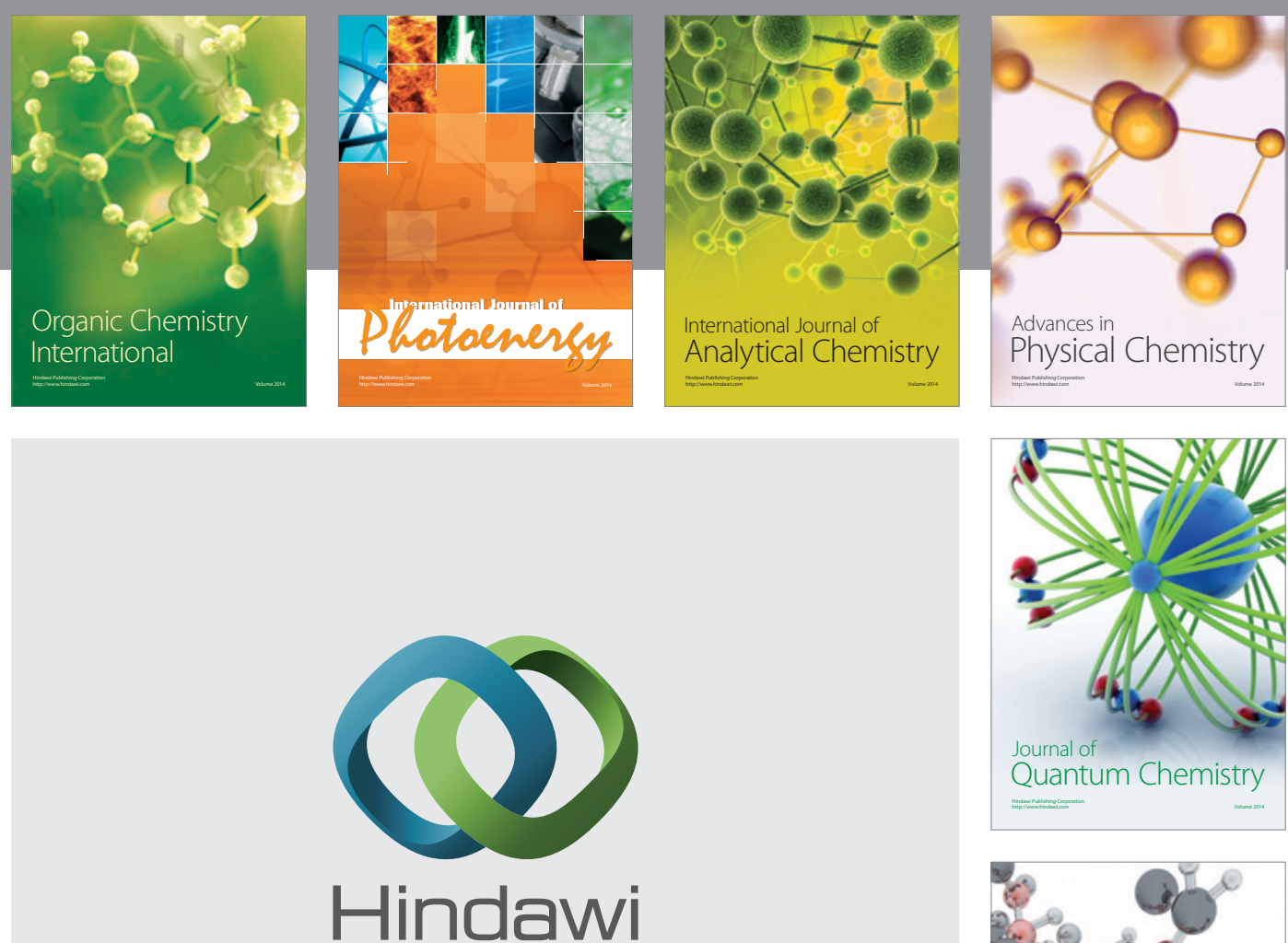

Submit your manuscripts at

http://www.hindawi.com

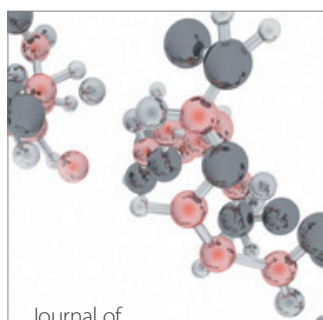

Analytical Methods

in Chemistry

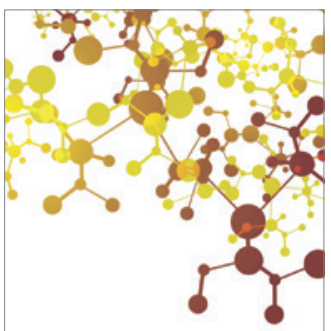

Journal of

Applied Chemistry

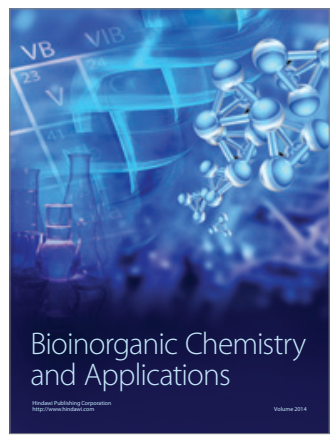

Inorganic Chemistry
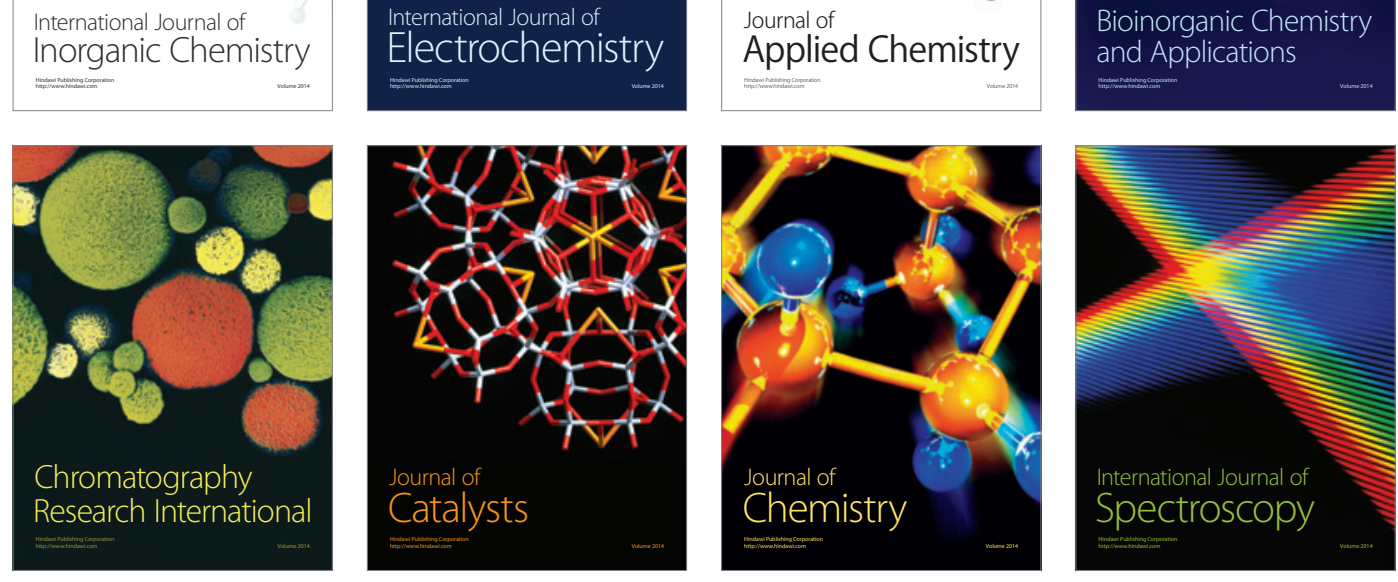